\title{
Helmut Ridder Neue Nachricht vom Aufhören des Verfassungsrechts nebst einem Vorschlag, wie Abhilfe zu schaffen sei*
}

Man könnte die Tragweite des strafgerichtlichen Einziehungsverfahrens betreffend den Entwurf eines Programms der Kommunistischen Partei Deutschlands, der am 8. Feb. I 968 auf einer Pressekonferenz in Frankfurt/M. der westdeutschen Offentlichkeit vorgelegt werden sollte ${ }^{1}$, zu unterschätzen geneigt sein. Denn am 22. Sept. 1968 hat sich eine neue westdeutsche kommunistische Partei (DKP) konstituiert, deren Orientierung und Programmatik ${ }^{2}$ jedenfalls nicht grundsätzlich aus dem durch vorausgegangene Emanationen der KPD abgesteckten Rahmen herausfällt und die dabei bisher vergleichsweise unbehelligt geblieben ist. Doch handelt es sich bei diesem Verfahren, dessen Schlußentscheidung der BGH nun durch sein Urteil vom I8. Feb. I9703 einer anderen Strafkammer des LG Flensburg überbürdet hat $t^{4}$ um mehr und Wichtigeres als eine dürâige juristische Randposse.

Unmittelbar politisch geht es um die Schnappmechanik der Mausefalle, in der die DKP konstituiert wurde: Solange sich das Illegalisierungsurteil des BVerfG gegen die KPD vom 17. Aug. I9565 mit den zu Recht oder Unrecht daraus gezogenen Folgerungen aktualisiert - und die definitive gerichtliche Anordnung der Einziehung des Programmentwurfs 68 wäre (ob mit dem Spruch des BVerfG

- Dieser Beitrag verfolge ein bescheidenes Ziel. Er kritisiert - grundgeseczimmanent und (angesichus der Qualitär des Objekes kaum hinreichend) polemisch - eine höchstrichterliche Entscincidung aus der rheinischen Provinz, die auch im Laht der vom atlantischen Lagerältesten amtlic verlautbarten neuen weltpolitischen Strategie einigermaßen begriffsstutzig erscheint. Er wiil verdeutlichen, daß sich aus der zu dieser Strategie auf nationaler Ebene zwangsläufig entstehenden offiziösen Ideologie Hilfen für die Liquidierung eines versteinerten westdeutschen Bürgerkriegs und fur die Verringerung des rechtsstaatichen Defizits gewinnen lassen. Sie zu nutzen, gebieter das in diesem Augenblidk partiell dedkungsgleiche und auf eine absehbare Wegstrecke hin gemeinsame Interesse beharrender wie verinderungswilliger Kräfte. Welche Moral dabei obzuwalten habe, mit weichen Mentaireservationen und Diversionsvorhaben und wo mit ihnen zu rechnen, was uber den ferneren Selbstauf der beim Wort genommenen Doktrin an Speikulation zulässig und was über die Verschiedenheit der stimulierenden MittelZwedk-Relationsvorstellungen schon jetzr feststellbar ist - von all dem handelt dieser Beitrag nicit.

1 Die tatsächlichen Vorgänge im Zusammenhang mit und in der Folge dieser Veranstalcung können wegen des breiten Presseechos als bekannt vorausgesetzt werden; vgl. im übrigen O. Schönfelds: Zwischenbilanz in W. Abendroth-H. Ridder-O. Sdiönfeldt: KPD-Verbot oder Mit Kommunisten leben?, rororo aktuell 1092 (\$968), S. เ7 ff.

2 Vgl. den am 23. Jan. 1969 auf einer Pressekonferenz in Düsseldorf vorgelegten Entwurf einer > Grundsatzerklärung der Deutschen Kommunistischen Parteik, abgedr. in Zeirgeschicht!. Dokumentarion, Sonderbellage zur Wochenzeitung • Blinkfuer «, Ausg. januar 1969.

$3_{3}$ SeR 2:69 I, Revisionsurteil des 3. Strafsenaes, BGHSe 23, 226, ausfuhrlicher abgedr. in NJW 70,318 .

- In der Vor- (ersten) Instanz harte die I. Große Strafkammer des LG Flensburg den Einzienungsantrag der Sraztsanwairschaft durch Urteil v. 30. 5. $1969-2$ KMs $_{4} / 69$ (I $113 / 68$ ) zurüdkgewiesen.

5 BVerfGE 5,85 ff. 
wirklich in Konkordanz oder nicht) ein höchst wirksaner Beitrag zu solcher Vergegenwärrigung -, läuft die DKP bei jeder ihrer Aktionen das Risiko, als "Ersatzorganisation « der "verbotenen " KPD von kurzer exekutivischer Hand zerschlagen zu werden. Sie, die trefflich auf "Verfassungsschutz « im Verstand des "Kalten Krieges« nach innen und nach außen eingespielte Exekurive, und nicht eine noch so namhafte, vielleicht gar ranghöchste, Vaterfigur an moraliscipolitischen Werbehimmel der Bundesrepublik, ist es, die im Zusammenwirken mit der seit nahezu zwanzig Jahren - um von den vorbundesrepublikanischen Hypotheken zu schweigen - auf die cinschlägigen Stercotypen eingearbeiteten Justizmaschine die Ǩlappe relativ autonom fallen lassen kann.

Der 3. Strafsenat des BGH hatte primär die in Literatur und Rechtsprechung nie explizit gestellte und sytematisch durchgearbeitete und von ihm auch jetzt nicht reflektierte Frage nach den substanticllen Auswirkungen eines "Parteiverbots" durch das BVerfG auf den Verhaltenspiclraum, und zwar sowohl auf die organisatorische Freiheit als auch auf die (individuelle wie kollektive) Handlungsfreiheit des Sozialsubstrats der »verbotenen " Partei zu beantworten. Seine Antwort ist womöglich noch massiver als alle bisherigen in Sachen KPD und Kommunismusverdacht ergangenen Judikate. Zentral auch für seine Entscheidung ist die in der Rechtsprechungspraxis des "politischen Strafrechts neuer Art «6 immer wiederkehrende wirkungsmaximierende Sentenz, "Sinn und Zweck "von Art. 2 I Abs. 2 GG sei es, »eine vom Bundesverfassungsgericit für verfassungswidrig erklärte Partei als Träger der politischen Willensbildung aus dem politischen Leben auszuschalten «'. A uf ihrer Grundlage vollzieht der Senat, nach landesüblichem Vorgang und wie auch der über juristische Formen und Fiktionen erhabene Soziologismus es nicht blinder vollbringen könnte, die "verbotene" Partei urbesehen mit ihren Mitgliedern identifizierend, die Exkommunikation dieser Mitglieder aus dem das ganze Bundesvolk umfassenden Verband der Träger staatsbürgerlicher Grundrechte. "Die für verfassungswidrig erklärte und aufgelöste KPD «, d. h. wiederum diese Gruppe von Staatsbürgern, für die es »keinen Raum politischer Handlungsfreiheit* mehr gebe, dürfe sich nicht einmal an der Diskussion über das "Verbot « der KPD beteiligen. Dieses Teilnahmeverbot stellt nichts anders als eine partielle Grundrechtsverwirkung für die davon $\mathrm{Be}-$ troffenen dar; es unterläuft mithin die dafür nach Art. I 8 GG verfassungsmäßig konstituierte Alleinzuständigkeit des BVerfG, worüber der Senat, ohne die (problematische) Stellungnahme des BVerfG zu dieser Frage zu erwähnen, mit souveränem Stillschweigen hinweggeht.

Die rechtsstaatlich-maßhaltend anmutende Verpackung des Verdikts in zwei lustig-listige scheinbare Einschränkungen läßt den tristen Befund in keinem andern Licht erscheinen. $\mathrm{Da}_{\mathrm{a}}$ wird einmal gesagt, das Teilnahmeverbor gelte, wjedenfalls solange der Spruch des Bundesversassungsgerichts bestehe «. Die KPD darf danach also, wenn sie einmal nicht mehr „verboten « sein sollte - solche Zukünfte visiert der Senat ungeachter der bundesweit herrschenden Auffassung von der perpetuierlichen Rechtskraft des "Verbots «, vielleicht mit einem Seitenblidk auf den unberechenbaren Gesetzgeber, immerhin an -, wenn auch nicht sicher (wer weiß schon im voraus genau, wie die konkrete Machtlage dann sein wird, und ob ein solcher Gesetzgeber wirklich materielle Finderungen hervor-

\footnotetext{
${ }^{5} \mathrm{Zu}$ dessen Gesamtwürdigung vgl. H. Copić: Grundgesetz und politisches Strafreche neuer Art (1967).

: Sie geht auf eine Formulierung des SRP-Urteils des BVerfG zurüdk: »... ist es der Sinn des veriassungssericintichen Spruches, dicse Ideen selbst aus dem Prozeß der politischen Willensbildun; auszuscheiden (BVerfGE 2, $x$ ff. [77]).
} 
rufen oder Liberalisierungsnebel verbreiten möchte), so doch möglicherwcise mit ihren Mannen in aller Offentlichkeit über Sinn und Unsinn des "Vcrbors « räsonnieren - ihr Pech halt, daß dieses Thema dann mangels Aktualität völlig witzlos geworden sein würde. Dann heißr es zum zweiten, die KPD dürfe sich an der Diskussion nicht durch Verbreiten eines »verfassungsfeindlichen « Programmentwurfs beteiligen. Daß dies indes beileibe nicht so zu verstehen sein kann, als ob die Vorlage eines verfassungsfreundlichen Entwurfs gurgeheißen würde, geht aus dem schon referierten generellen Ausschluß hervor, wonach die »aufgelöste Partei « überhaupt nichts tun, so auch nicht etwa für die NATO werben oder jenseits der sie bewegenden Legalitätsfrage »in irgendeiner Form staatsbürgerliche Aufklärung ... betreiben« darf. Alle Ausführungen der Entscheidung zum Entwurfsinhalt, desgleichen die Bemerkungen über die Werbewirkung der Veröfentlichung des Entwurfs für die KPD (sollte sie etwa verpflichtet sein, Propaganda gegen sich selbst zu betreiben?), verschleiern nur, daß der Senat über die der KPD entstammenden Kommunisten (die sich wohl nicht gerade in NPD-, CSU- o. ä. Ortsgruppen umgründen werden, um in den unangefochtenen Genuß kollektiver Außerungsfreiheit zu gelangen) wieder das absolute, totale und unbefristete politische Schweigegebor verhängt (das freilich durch die Tärigkeit der DKP ridikülisiert wird) und erneut den Innenministern der Bundesländer noch nach fast drei Jahren ihre KPD-Empfehlungen vom r2. Oktober 1967 um die Ohren schlägt (mit denen der Weg für die - notwendig mit der Vorlage eines neuen Programms verbundene - Neukonstituierung einer kommunistischen Partei freigemacht werden sollte $\left.e^{8}\right)$.

Der nicht solchermaßen disqualifzierten und zum Glück aus richtigen Menschen bestehenden, mithin eventueller solidarischer Symparhien würdigen Offentlichkeit sieht der Senat es im übrigen nach, daß in ihr über das Verbor der KPD diskutiert wird. Sein Mißbehagen über diese Einbuße an Konformismus ist freilich unverhohlen und äußert sich in aufschlußreichen Korrekturen an zutreffenden und unbestreitbaren Feststellungen der Vorinstanz. Hatte das LG Flensburg noch "Stimmen in der Offentlichkeit, sogar von maßgeblichen, im politischen Leben stehenden Personen « vernommen, "die eine Auseinandersetzung mit den Zielen der KPD suchen«, so vermochte der BGH nur noch »hier und da«, $d$. h. allemal unrepräsentative Diskussionen wahrzunehmen; und aus den landgerichtlichen "so vielen Jahren « nach dem für die Entwicklung des politischen Systems der Bundesrepublik so entscheidend wichtigen KPD-Urteil des BVerfG werden ihm bagatellisierte »einige Jahre " des Zurückliegens jener zu einem Hauptfaktor der Transformation gewordenen "Eselei mit besonders langen Ohren " (Adolf Arndt).

Die Wirklichkeit in allen ihren Erscheinungsformen, nicht zuletzt als "Verfassungswirklichkeit», hat auf die insofern stets "progressive", von Legitimationsproblemen und sonstiger politischer Theorie hingegen kaum beschwerte deutsche Jurisprudenz immer eine magische Anziehungskraft zulasten des Gesollten, aber nicht Verwirklichten ausgeübt; nach Maßgabe der Solidität ihrer machtmäßigen Befestigung hat sie es bereits vor einem halben Jahrhundert sogar bis zur »normativen Kraft des Faktischen«, d. h. bis zur Nullifizierung der Norm, gebracht. Doch wenn noch gänzlich unbefestigte Wirklichkeit, in den konstitutionell ge-

8. Der Ulmer BeschluB [der Innenminisecr $v .12 .10 .67$ ] birge doch wohl die Konsequenz, man werde zunädist... abwarten, um das Verhalten der newen politiscien Kräfte zu prufen *, meinte denn auch sehr richtig das Blatt für die Klugen Köpie am Morgen raci dem enervierten Polizeicoup gegen die Programm-Pressekonferenz in Frankfurt (FAZ v. 9. 2.68: -Aufgelöste Pressekonferenze). 
währleisteten Frei- und Innovationsräumen gebildet, aus dem Dunkeln ins Licht zu treten sich crdreistet, wird sie auf das - unrichtige - Maß der Einflußlosigkeit zurückgeschnitten, das die Macht ihr belassen will. Zwar läbt auch der 3. Strafscnat des BGH keinen $Z$ weifel mehr daran, daß staatsbürgerliche Aufklärung "nicht etwa ein Vorrecht der [gleichwohl dafür] zuständigen [!] Behürden « ist. Aber cigentlich müßte sie seines Erachtens nur von "Institutionen « geleistet werden, wozu er die via facti »zur Information berufenen Publikationsorgane wie Presse, Rundfunk, Fernsehen und nicht zuletzt die politischen Parteien selbst « zählt. Dieser Minimalanforderung an das Etabliertsein könnten, streng genommen, schon die »hier und da (d. h. auch: wahrscheinlich und hoffentlich erfolglos) jenseits des Themenkatalogs der in öffentlicher und privater Hand gerierten Bewußstseinsindustrie stimulierten Normalbürger nicht genügen. Art. s GG, vom demokratischen Zentral- und Fundamentalrecht zum exzeprionellen Gnadenerweis aus "Toleranz « für Nichtkonformisten degeneriert, schmuggelt sie indes gerade noch durch. Die agitatorisch versierten und potentiell effizienteren KPDMitglieder dagegen passieren in keinem Fall. Die Entinstitutionalisierung ihrer Organisation durch den Spruch des BVerfG schwemmt auch ihren individuellen Grundrechtsschutz mit hinweg. Wo käme man denn sonst hin!? Vielleicht gar zu Einsichten in die prinzipielle Ungeschiedenheit von »bürgerlicher" und »proletarischer Revolution «, die zwar einem Lenin-Gedenkjahr gut anstehen würden, aber bei allen konvergenztheoretischen Sympathien doch nicht richtig sein können, weil sie nicht richtig sein dürfen?

"Nun« ist aber, wie der Senat auf Kosten der Logik weiter lehrt, »zweifellos für die Aufklärung des Staatsbürgers, von dem eine möglichst umfassende politische Urteilsbildung erwartet wird und der insbesondere auch für die geistige Auseinandersetzung mit dem Kommunismus - im Interesse der Erhaltung der Demokratie - gerüstet [!] werden soll, die Kenntnis und das Studium gerade der neueren komunistischen Programme und Grundsatzerklärungen von Wert火. Er fordert damit nicht weniger als eine Urteilsbildung, die einer schon anderweit erfolgten Urteilsbildung über denselben Gegenstand verpflichtet ist; das »insbesondere " in dieser Deklamation verbesondert nichts und mitnichten, sondern leitet die Aufhebung der Forderung nach Urteilsbildung ein. Postuliert wird nicht Urteilsbildung, sondern politisch-moralische Aufrüstung, die den Staatsbürger beähigt, jederzeit rückhaltlos für die Erhaltung der "Demokratie« einund gegen jede Veränderung des mit ihr identifizierten status quo der Verhältnisse aufzutreten. - Was sich tun ließe, um dem nachzukommen, kann sehr einfach beschrieben werden. $\mathrm{Da}_{\mathrm{a}}$ die völlig freie Erfindung nicht authentischer "neuerer kommunistischer Programme und Grundsatzerklärungen immerhin heikel wäre, vertrauen die bis zum Tage $X$, an dem der Bannspruch des BVerfG zu »bestehen « aufhört, sehr passend vom Meinungs- und Informationskonzert ausgeschlossenen KPDisten ihre authentischen Programmvorstellungen den »Institurionen «, etwa den andern Parteien oder dem Bundespresseamt, an. Diese (würden sich das Material übrigens mit Hilfe der bestehenden Feindbeobachtungsbehörden sowieso verschaffen; und sie) werden, da sie sich, schon weil vorhanden, nicht partikularen Interessen, sondern bloß dem gemeinsamen Besten verpflichret fühlen, bei der Veröffentlichung nicht verfehlen, durch Auswahl, Präscntation und beigefügte eigene Annotationen die Meinungs- und Urteilsbildung des Publikums zu erleichtern und zu objektivieren; auf die mobjektive" Aufklärung der Staatsbürger kommt es dem BGH ja gerade $a n^{2}$. Das Rezept ist

- Ein instruktives Beispiel sorgender sObjekrivierung \& bieter die Schrift von C. H. Boetrcher: Eine neue KPD? (196S), die es naci einigen hundert Seiten Einstimmung wagen kann, 
alt und ausweislich der Geschichte probat: Als der reformatorische Unfug der individuellen Bibellektüre nicht mehr einzudämmen war, hat die römische Kirche ihre Gläubigen noch generationenlang durch das Exklusivprivileg der von ihr approbierten und annotierten Textausgaben gegen ein vergleichsweise viel gefährlicheres hochexplosives Gemisch sozialrevolutionärer Giftstoffe zu schützen und im Kampf zwischen Botschaft und Welt die Welt in ihrer ganzen hierarchischen Wirklichkeit hervorragend zu stabilisieren vermocht.

Diese Bevormundungs- und Immunisierungsstrategie verträgt sich freilich nicht damit, daß das Grundgesetz u. a. in Art. s staatsbürgerliche "Mündigkeit« bereits voraussetzt. Der Senat konnte m. a. W. nicht darüber hinweggehen, daß die von ihm vorgeschriebene scheuklappenperspektivische "Mündigkeit « und die grundgesetzlich-axiomatische Mündigkeit sich gegenseitig ausschließen. Diese Schwierigkeit bei der justiziellen Förderung der Produktion von »Mündigkeit« im ersterwähnten Sinn geht er mit der milden Feststellung an, daß »die Einziehung der Druckschriften die in Art. ร Abs. I GG gewährleisteten Grundrechte der Meinungs-, Informations- und Pressefreiheit ... berührt [!] «. Damit ist die besorgniserregende Frage impliziert, ob $\$ 86 \mathrm{StGB}$ ganz oder teilweise gegen den nach dem rechtsstaatlichen Verständnis von der Vorrangigkeit des Verfassungsrechts stärkeren Art. 5 GG verstoßen könnte. Die Besorgnis legte es dem Senat nahe, sich eines Instruments zu bedienen, dessen vorzügliche Eignung zur angemessenen, d. h. ohne riskante Erzeugung von allzu ungebührlichen Selbstbindungen pro futuro in jedem konkreten Einzelfall das $\mathrm{MaB}$ des staatspolitischen Erforderlichen erreichenden (und dabei die abstrakt-normative Verfassungssilhouette überhaupt nicht beeinträchtigenden) Dezimierung von störenden Grundrechten erwiesen ist, das seit dem Lüth-Urteil des BVerfG ${ }^{10}$ auch in Literatur und Rechrsprechung zum Verfassungsrecht sehr schnell seine heute kaum noch angefochtene Heimstatt gefunden hat und den Gerichten das ermöglicht, was das BVerfG nur wenig früher mit vollem Recht dem Gesetzgeber (dessen Tätigkeit freilich auch stets die normative Silhouette verändert) untersagt hatte: $\bowtie$... in einem circulus vitiosus die vom Grundgesetz bereits getroffene politische Entscheidung in die Hände des einfachen Gesetzgebers [Gerichrs] « zurückzuspielen und Grundrechte ihrer »rechtlichen Bedeutung « zu »entkleiden «11. Kurzum: Der 3. Strafsenat des BGH benutzt, wie vor ihm in derselben Sache schon die I. Große Strafkammer des LG Flensburg und in zahllosen anderen Grundrechtssachen andere Greichte, das zivilistische Vehikel der "Güterabwägung «.

Die in den Urteilsgründen der Grundrechtsrechtsprechung sich ereignende "Güterabwägung «12 ist zumeist ein Vorgang, bei dem zwar die allegorische Waage der Justitia bühnenwirksam mit Flutlicht angestrahlt, aber durchaus nichts mehr abgewogen wird. Steht das "der vom Grundgesetz bereits getroffenen politischen Entscheidung" entsprechende Ergebnis objektiver Rechtsanwendung (Verfassungsbefragung) quer zu den Verhältnissen und dem von ihnen geprägten justiziellen Vorverständnis, so wird durch die Inszenierung der »Güterabwägung “

den Leser einem (mit umsidtigen. $Z$ wischenüberschriften versehenen) Auszug aus dem Entwurf 68 auszusetzen (S. 13 I ff.).

10 BVerfGE 7, 198 f.

"11 BVerfGE ;, 225 ff. [240].

12 Sie tritr in mannigfachen Konstruktionsvarianten auf und zcitigt von der holzschnittartig einfachen Erfindung simmanenter Grundrechtsshranken $z$ wischen reputierten (und daher schützenswerten) und etwas weniger reputierlichen (und daher zu vernachlässigenden) Grundrechtsträgern (grundlegend BVerfGE 7, I98 ff. v. Is. x. I958 gegen BVerfGE 7,230 ff. v. 15. I. I958) ein von den bisherigen theoretischen Bemühungen um die bundesrepublikanische Praxis der $\rightarrow$ Rechtsgewinnung \& noch nicht vermessenes hödist abwechslungsreiches Landschaftsbild einer höchst einheirliden politischen Vegetztionsebene. 
nur die in foro interno bereits vollzogene Umwertung der Verfassungswertung in das Judikat umgesetzt. Sonst wäre die Veranstaltung ja auch überflüssig und würde nicht stattfinden. Doch wird der Nacbvollzugscharakter der "Güterabwägung" nicht transparent, weil sie in zwei Argumentationsschüben zur Darstellung gelangt, die, obwohl von sich fortsetzender Gleichsinnigkeit der Bewegung, durch eine im beziehungsreichen Waagenbild Offenheir der "Spannungslage" assoziierende $Z$ wischenstation voneinander getrennt sind. Im ersten Schub rückt das aggressive unterverfassungsmäßige "Gut " zur "Gleichwertigkeit " mit dem Grundrechtsschutzgut auf, und erst nach mehr oder minder ausführlichem Umkreisen des dadurch kreierten "Spannungsverhältnisses « mit mehr oder minder zahlrcichen Andeutungen der Offenheit der "Lösung « in abstracto erfolgt dann im zweiten Schub der endgüitige Rangaustausch in concreto. Der nun erfolgt gewiß; denn das ist - siehe oben - die einzige (selten bewußte) ratio des Spektakels.

Im vorliegenden Fall haben beide Gerichte Art. 21 Abs. 2 GG zur Anhebung von $\$ 86 \mathrm{StGB}$ auf die Verfassungsebene benürzt, um sich jeder Prüfung der Frage, ob $\$ 86 \mathrm{StGB}$ etwa ein besonderes (nicht "allgemeines«) Gesetz im Sinne von Art. $\varsigma$ Abs. 2 GG sein könne, zu entziehen. Weil $\$ 86 \mathrm{StGB}$ min enger Anlehnung [!] an Art. 2I Abs. 2 GG ebenfalls den Schutz der freiheitlichen demokratifchen Grundordnung gewährleisten soll«, partizipiert er selbst am Vorrang der Verfassung; die Intention heiligt das Gesetz. In der Tat - das muß schon immer die Maxime des BGH im politischen Strafrecht gewesen sein, da er die Verfassungsmäßjigkeit selbst der monströsesten Exzesse dieses Gesinnungen, Meinungen und Kontakte hysterisch kriminalisierenden »Rechts « eines perpetuierten partiellen Ausnahmezustands nicht einmal dann auch nur einen Augenblick in $Z$ weifel gezogen hat. wenn ihm keine als containerlift geeignete Bestimmung des Grundgeserzes einfiel. Und Art. 2 I Abs. 2 selbst gilt dem Senat als mit Art. 5 Abs. I GG nicht nur gleichrangig (was eine Banalität ist), sondern auch als im "Verfassungsrange gleichwertig [!] «, worauf der von Art. 2I Abs. 2 GG umhüllte $\$ 86 \mathrm{StGB}$ zwecks konkreter "Lösung « des schaukämpferischen "Konflikts « mit Art. s GG weiter zur Uberwertigkeit aufsteigen kann. Es ist keine emorionelle Ubersteigerung, wenn man diese Gleichwertigkeitsproklamation als eine skandalöse Preisgabe der Grundlagen liberaler (»westlicher «) Demokratie bezeichnet. Denn heute wie früher - und auch unter dem Grundgesetz, das diese Grundlagen nicht preisgeben wollte - sind Meinungs-, Informations- und Pressefreiheit für diese Verfassungsordnung konstitutiv und als konstitutive Normalität von entsprechenden Grundrechten gewährleistet, wohingegen die Illegalisierung politischer Parteien ein systemfremdes, politisch frag würdiges, außerordentlich ambivalntes und mit äuferster Restriktion zu handhabendes, andernfalls, wie das Trauerspiel des mehr als fünfzehnjährigen justiziellen Bürgerkriegs gegen die kommunistische Minderheit des Bundesvolkes erweist, systemzerstörendes Institut des Ausnahmerechts ist ${ }^{13}$.

Das IG Flensburg hatte nicht ohne einen Hauch von realistischem Gespür fürr gewisse norwendige Veränderungen in der bundesrepublikanischen Europa-Politik denn auch mit $\$ 86$ Abs. 3 StGB eine Hintertür entdeckt, durch die es selbst den Fahrkorb zur "Güterabwägung " auf Verfassungsebene noch in der Ruhestellung wieder verlassen konnte, und sogar sehr entschieden ausgeführt: "Es macht ... das Wesen der Demokratie aus - und das kommt in $\$ 86$ Abs. 3 StGB zum Ausdruck -, daß sie von der freien Auseinandersetzung verschiedener sich widersprechender politischer Meinungen lebt." Die gewiß sehr sensible Antenne

13 Hierzu aiigemein H. Ridder: Grundgesetz, Notstand und politisches Strafreche (rg65). 
des BGH blieb dagegen auf den Empfang schon beendeter amtlicher Sendungen eingestellt, die sich schon länger keines atlantischen Beifalls mehr erfreuen konnten und zur wachsenden Isolierung der Bundesrepublik auch im Lager ihrer außenpolitischen Bündnispartner beigetragen haben; hier mag nachholende Lektüre quasi-offiziöser Verlautbarungen hilfreich $\operatorname{sein}^{14}$.

Wahrscheinlich hat sich die flensburger Strafkammer, die sich mit dem am Ende von ihr auch verwirklichten Bekenntnis zur freien Auseinandersetzung der politischen Meinungen als Wesenselement der Demokratie so deurlich von der Front des inneren "Kalten Krieges « abzumelden angeschickt hat - ohne sich des Widerspruchs zur vorher angewandten "Güterabwägung" im Kontext "streitbarer Demokratie" voll berwußt zu werden-, allerdings Tüuschungen über die Intention der Urheber von $\$ \$ 6$ Abs. 3 StGB hingegeben. Soweit diese Vorschrift nicht bloß als "staatsbürgerliche Aufklärung " bezeichnete antikommunistische Indoktrination normativ profilieren sollte, kann sie höchstens als partikuläres Toleranzedikt innerhalb eines die demokratische Normalität überwuchernden Ausnahmerechts entworfen sein und begriffen werden, womit sie das "Wesen der Demokratie" noch weiter verfehlt als die nach dem Vorgang des Lüth-Urteils so häufig strapazierte rückwärtige Bestreichung in concreto grundrechtsaffizierender einfach-gesetzlicher Normen mit dem Lichtkegel des Grundrechts.

Der BGH hat denn auch nicht verabsäumt, nachdem die Strafkammer $\$ 86$ Abs. 3 StGB als Ausprägung des Grundrechts der Meinungs- und Informationsfreiheit bezeichnet und damit der nunmehr zur Entscheidung aufgerufenen landgerichtlichen Schwesterkammer einen möglicherweise prakrikablen (Um-) Weg zur Wiederherstellung eines streitbarkeitsfreien demokratischen Verfassungsverständnisses gewiesen hat, aus den Materialien zu $\ 86$ Abs. 3 StGB, nämlich dem schriftlichen Bereich des Bundestags-Sonderausschusses für die Strafrechtsreform, darzutun, daß es hier nur um die Straffreiheit *sozialadäquater Benutzung... z. B. zu wissenschaftlichen oder künstlerischen $Z$ wecken " gehe. So gewinnt er die seit dem Ende der dreißiger Jahre für eine progressive Anpassung des recht-

It Sehr förderlici erwa die klarsichrigen und zielklaren Darlegungen der Parl. Suanssekreririn beim Bundeskanzler, Dr. Katharina Focke: Europa-Politik nach Den Haas. Europa-Archiv 25, 8. Folge (v. I .4 .70 ), S. 267 ff.: Un $_{\text {m }}$ als exemplarische Ordnung auch für Gesamteuropa Wert zu haben, muß die [Europäische] Gemeinschaft von jeglicher antikommunistischer Kreuzzugsideologie ebenso wie von allen Roll-badk-Anklängen der „Kalee-Kriegs-Zeit* freibleiben. Es gibt viele Menscien, die in den fünfziger Jahren die Gemeinschaft im wesentichen als eine antikommunistische Unternehmung betrachtet hatten. Heute sollte aber immer wieder darauf hingewiesen werden, daß die Gemeinschaft niche eine Anti-Gemeinschaft ise, sondern daß sie eine Pro-Gemeinschaft ist, eine Gemeinschaft für den Frieden, für eine Rechrsgemeinschaft in Europa (S. 26S). Was die innigen Zusammenhänge einer solctien Politik mit dem Ausbau einer Position der sozialen Stärke durch -Verbesserungen der Funktionen unserer eigenen Gesellschaften * (oder innere Reformen $\alpha$ ) im Rahmen weltweiter Uberlebensstrategien

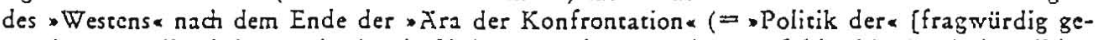
wordenen] militärischen und wireschaftlichen -Srärkea) angeht, empfiehlt sich ein eindringliches Studium der Ansprache Sr. Exzellenz, des Botschafters der Vereinigten Stanten in der Bundesrepublik Deutschiand, Kenneth Rush, vor der Deutscien Geseilschaft für auswärtige Politik v. 10. 3. 1970: Eine Fra der Verhandlungen - Zielserzungen und Erfordernisse, deren schriftlidhe Fixierung (hektogr.) die amerikanische Botschait dem Veranstalter des Vortragsabends abgenommen hat (Embassy of the United States of America - United States Information Service, Bonn-Bad Godesberg). In der Gartenlaube der Anspruchsvollen reden freilich vorerst wieder nur Gäste dem $\rightarrow$ friedlichen Wettstreit der Systeme (zu dem auch die KPD einiges zu sagen hat) das Wort (M. Kriele: Der Streir um die Ostpolitik, FAZ Nr. I so v. 3. $7.70=$ Bulletin des Presse- und Informationsamtes der Bundesregierung, Nr. 92 v. 8. 7. 70, S. 909 ff. [unter Auszeichnung als Sonderdrudk und Zitat des FAZ-Vorspruchs: • jenseits der parteipolitischen Auseinandersetzung a)], während ihre haupramtichen Kustoden, so sie nictic obdachlos werden wollen, cher auf die besonderen und vielfach liebenswerten Eigenschaften * aufmerksam machen, die szum Beispiel den russischen Menschen im Sowjerreich eigen sind * (N. Benciniser: West und Ost, FAZ Nr. 172 v. 29. 7. 1970). 
lichen Sollens an das soziale Sein entwickelte, an lebensfremd konstruierten * Fällen « als lebensnah plausibel gemachte, in anderen Disziplinen wohlerprobte und an schlichter Handlichkeit der „Güterabwägung« weit überlegene Allzwedkformel der "Sozialadäquanz " als »ein allgemeines Prinzip, dessen Bedeutung sich auf das Strafrecht beschränkt, sondern die ganze Rechtsordnung erfaßt«, auch dem Verfassungsrecht - ein markanter Höhepunkt in dem rechtsfeindlichen Prozeß der Zerstörung der demokratisch-partizipatorischer Grundrechte des Grundgesetzes.

Der KPD würde es danach nicht helfen, wenn sie den Entwurf 68 vertonen und von einem gemischten Chor vortragen ließe. Das wäre zwar künstlerisch, aber, abgesehen davon, daß es wegen der damit verbundenen Werbung nicht als "sozialadäquat " betrachtet werden könnte - kommunistische Werbung kann laut BGH nie "objektive Aufklärung « sein, wie sie von den übrigen Parteien laufend erbracht wird -, wäre es auch nach den Mutmaßungen vieler billig und gerecht denkenden Zeitgenossen »in strafrechtlicher Hinsicht« nicht "gänzlich unverdächtig" und auch von daher nicht »sozialadäquat «. Die NPD hinwiederum brauchte bei der Veröffentlichung von KPD-Programmen keinen künstlerischen Aufwand zu treiben, weil sie als $* z u r$ Information berufenes Publikationsorgan" eo ipso staatsbürgerliche Aufklärungsarbeit leistet. So kann die Einbringung der "Sozialadäquanz " einerseits die Gewinnung der staatspolitisch notwendigen Ergebnisse nicht gefährden. Sie beugt aber andererseits in einer bedeursamen Verfeinerung, die der BGH ihr bei der Ubernahme ins Verfassungsrecht angedeihen läßt und die zugleich eine wesentliche Vereinfachungshilfe ist, allfälligen neuen Pannen beim Betrieb der "Güterabwägung "fern der Residenz des Rechts vor. "Soweit« nämlich "politisches Handeln in Betracht konmme «, macht der Senat den Raum der msozialen Handlungsfreiheit» vorsorglich nicht von der Billigung durch die möglicherweise doch nicht standfeste $*$ Allgemeinheit " abhängig, sondern läßt die verbindlich von dem - seinerseits von der ausnahmerechtlichen Bestimmung des Art. 2 r Abs. 2 GG inspirierten - einfachen Gesetzgeber ausgedeutete "verfassungsmäßige Ordnung der Bundesrepublik Deutschland " die "Allgemeinheit " vertreten. "Soweit politisches Handeln in Betracht kommt«, wird die Verfassung somit nicht nur für den Einzeifall, sondern allgemein unter das Gesetz gestelit, so daß die Waage der "Güterabwägung " künttighin nicht mehr in jedem Einzelfall aus dem Requisitenschrank hervorgeholt werden muß. Der Rationalisierungseffekt liegt auf der Hand. Die Stabilisierung der mit der "verfassungsmäßigen Ordnung identifizierten Gesetzgebung durch die Bindung an sich selbst ist der unverkennbare Erfolg dieser Preisgabe der Rechtsstaatlichkeit. Und trotzdem kann, da das alles nur durch ein - Berücksichtigen « der »verfassungsmäßigen Ordnung " bewerkstelligt wird, falls der Gesetzgeber das gemeine Beste einmal aus dem Auge verlieren sollte, die Vertretung der Allgemeinheit doch zwanglos wieder vom $B G H$ übernommen werden. Verfassungen kommen, Verfassungen gehen...

\section{II.}

Die Durchführung des Illegalisierungsverfahrens gegen die SRP, die als unmittelbare Fortsetzung der aufgelösten NSDAP15 gar nicht erst in den Genuß

15 Was das BVerfGE einleitend vorsichtig und summarisch nur als Ahnlichkeiten mit der NSDAP (BVerfGE 2, 27) bezeidnet, ist nach dem Ergebnis der Bewcisaufnahme (a. 3. O. 27-75) weit mehr, weswegen denn am Ende auch bereits von - Wesensverwandeschaft (a. a. 0 . 74) und Nachfolgcorganisatione (a. a. O.73) gesprodien wird. 
des formellen Parteienprivilegs nach Art. 2 I Abs. 2 GG hätte gelangen dürfen ${ }^{16}$, war nicht nur ein meisterlicher Beitrag zur Wiederherstellung der das Rechts- = Linksradikalismusklischee zementierenden Denkbehinderungen ${ }^{17}$. Sie stellt auch eine beispielhaft ingeniöse Nutzung eines geschichtlichen Augenblicks dar, mit der einer die Geschichte verfehlenden, im wahrsten Sinne des Worts reaktionären Entwicklung Aufwind verschafft wurde.

Das BVerfG des Jahres I952 konnte - am noch frühen Morgen nach der Zertrümmerung des nazistischen Gewalt- und Herrschaftsapparats durch die antifaschistische Weltbürgerkriegskoalition - kaum dem Verfassungsgebot demokratischer Parteilichkcite untreu werden; und das heißt: Wenn schon im Verfahren nach Art. 2 I Abs. 2 GG gegen die SRP vorgegangen wurde, so war es zweifellos der Sinn seines konkreten Spruches gegen die SRP, ndiese Ideen [ = die Ideen der SRP, die unmodifizierte NS->Ideen، waren] selbst «, die auch über "Weltanschauung " und andere afterwissenschaftliche Doktrinbildung hinaus nicht auf Wissenschaft hin sublimierungsfähig sind, waus dem Prozeß der politischen Willensbildung auszuscheiden «. Den Akteuren dieser - wie sehr bald zutage trat - Einübung sowohl in Antikommunismus als auch zugleich in Antiparlamentarismus war dabei nur zu geläufig, daß Gerichte, obwohl sie jeweils nur den konkreten Fall rechtsverbindlich entscheiden und zu entscheiden haben, um der (aufgrund der spezifisch deutschen historischen Hypothek wurzeltiefer Trennung von Demokratie und Rechtsstaat unpolitisch-) rechtsstaatlichen Überzeugungskraft ihres Spruchs willen generalisierend begründen zu müssen glauben, hier also das BVerfG sich über die Wirkung von Illegalisierungsentscheidungen gegen politische Parteien schlechthin auslassen würde. So wurde das SRP-Urteil zum Bahnbrecher des um seine antifaschistische Funktion gebrachten und antikommunistisch umfunktionierten, den politischen Prozeß von links aufrollenden verfassungswidrigen Maximalismus, dessen Tendenz während des KPD-Verfahrens vor dem BVerfG auch ungeniert offenbart wurde ${ }^{18}$, der die

10 Daher - und weil er die Systemfremdheit des Illegalisierungsinstituts verkennt - kann W. R. Beyer: Das Karisruher Bundesverfassungsgeriche und die KPD, Staat und Reche is (1969), S. 76 ff., nicht gefoigt werden, wenn er meint, Repressionsobjekt des Art. 21 Abs. 2 GG seien sauschließlich die Parteien, die an der Wiege des Bonner Staates durch die Kontrollratsgeserzgebung vernichter. im Keime aber noch nicht ganz erloschen waren (S. SI). Zur Verhinderung ihres Wiederaufkommense hätre es, mögen manche Verfassungsväter es auch für notwendig gehalien haben, des Art. 2I Abs. 2 GG gar nicht bedurft. Zutreffend ist dagegen die anschließende Feststellung Beyers, es sei unmöglich, daß eine der an der Schöpfuns des Grundgesetzes (wenn auch durch Gegenstimmen) beteiligten Lizenzparteien selbst unter die illegalisierbaren Parteien fallen könne. Daraus (oder auch aus anderen Umständen) auf die >torale Nichtigkeit des KPD-Urteils zu schließen, ist freilich ohne prakrikable Konsequenzen.

17 , Das Maß des Erträglichen a war nach $R$. Schuster ,überschrittena, als zwedss Verfestigung des ins Zwielicht geratenen $>$ KPD-Verbots a der Bundesgeschäftsführer der SPD, H.-J. Wisósnewski, den auch weiter links stehende denkunfreudige Gemüter beeindrudkenden (heute wohl überholien) Versuch unternahm, sdie schiefe Oprik dadurch zurechtzurüoken, daß dem KPDVerbot nun ein NPD-Verbot entgegengestellt wird, das, wenn es auf der heutigen grundgescezlichen Basis niche zu erwirken sei, notfalls durch eine Inderung der Verfassung erzwungen werden sollte (Demokratie - nur im Sonntagsanzug? - Die Daseinsberechtigung extremer Parteien, DIE ZEIT, Nr. 48 v. 29. 1 r. 1968, S. 32). Dem Autor, der sid bereits kurz zuvor reche ausführlich und in richtiger Erfassung des Ausnahmecharakters des Instituts der Parteiillegalisierung Uber die Grenzen der sabwehrbereiten Demokratiec๙ geäußert hatte (JZ 68, S. I\$2 ff.), wird die nach US-Präsident $R$. Nixon unvermeidlid gewordene weitweite Wende von der - Konfrontation a zur Kra der Verhandlungen a nunmehr vielleidh aud die hilfreiche weirerführende Erkenntnis zuwachsen lassen, daß es keine sobjektiven Bedingungen des Arr. 2 I II GG gibt, >die einen Untershied von links und rechts nidht kennen und nidit kennen dürfen (a. 2. O. S. I59), und daB - eine derartige prinzipielle Differenzierung a keineswegs norwendigerweise willkürlich und damit eine Verletzung des Gleidheitssatzes sein wurde* (ebd.). Der Faschismus jedenfalls ist nach Auschwitz, wie die s X̆ra der Verhandlungen a als Geschichtsiexikon mit freilidh bedaueriicher Verspätung verdeutlidht, kein Partner und kein Gieidberechtigter.

${ }^{18}$ Ith habe literarish mehrfach (vgl. etwa: Aktuelle Rechtsfragen des KPD-Verbots [1966], 
im Prominentenmund geläufige Wendung, auch Mitglieder der illcgalisierten KRD seien »selbstverständlich» hinsichtlich der Wahrnehmung ihrer staatsbürgerlichen und politischen Grundrechte in keiner Weise disqualifiziert, als hohle

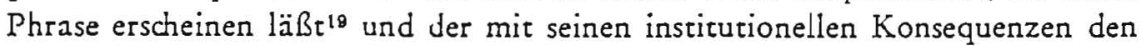
Parlamentarismus aus den Angeln hebt ${ }^{20}$.

Dem ruinösen Verschleiß an demokratischer Substanz ${ }^{21}$ hat das KPD-Urteil des BVerfG nun zwar durch manche dem Zeitgeist verpflichtete schnelle Ubernahme objektiv Vorschub geleistet?2. Dem stehen aber entscheidend wichtige, bisher kaum beachtete und noch weniger gegen den Selbstlauf des dem SRP-Urteil entnommenen wirkungsmaximierenden Leitsatzes fruchtbar gemachte Abstriche gegenüber, die die Feststellung rechtfertigen, daß das BVerf $G$ auch das KPD-Urteil nicht im Himmel der Geschichtslosigkeit gefällt hat. So tauchte, wenn auch verdunkelt, abgeschwächt und noch innerhalb einer umgestülpten Rangordnung, die im SRP-Urteil verständlicherweise - nämlich weil der SRP (=NS)-Ausschluß keine Betätigung der Notbremse nach Opportunität, sondern Bestätigung des Fundaments des Grundgesetzes war - gar nicht enthaltene Erkenntnis der Zugehörigkeit der Parteillegalisierung zum Regime des Ausnahmezustands in der Feststellung auf, daß das Institut des Art. 2 I Abs. 2 GG nicht dem "Mindeststandard « freiheitlicher Demokratie anzurechnen sei.

Dies geschah übrigens in Verbindung mit der Selbstbefristung der Geltungsdauer des Urteils im Hinblick auf eine deutsche "Wiedervereinigung «, einem weiteren einschränkenden Moment, das bisweilen schon erörtert worden ist ${ }^{23}$. $\mathrm{DaB}$ die mit "Wiedervereinigung «. bezeichnete Herstellung einer gesamtdeutschen staatlichen Einheit unter dem Druck der Weltlage nun auch förmlich aus den Agen-

S. 44 f. m. Anm. 82) auf solche offenherzigen Eingeständnisse hingewiesen, nach denen das Verianren gegen die damals schon wegen ihrer Winzigkeit nicht s gefihrlichex KPD primär in Gang gesetzt worden ist, um als slinksradikala gebrandmarkte ideelle politische Rüume zu sterilisieren.

19 Die Wirklichkeit läßt sid zu einem nicht unbeträdthliden Teil an der Rechtsprechung auf dem Gebiet des politischen Strafrechts erkennen, und diese spiegelt, allerdings mit den schon vermerkten gelegentliden Verspätungen, den audh behördlich verlautbarten Trend. So korrespondiert das vorliegende BGH-Urteil mit der faktisch amtichen Erklärung, ^daß auch dic Verbreitung eines neuen Programms einer verbotenen Partei strafbar sei, wobei es $>a$ uf den Inhalt dieses Programms und scine Verfassungswidrigkeit überhaupt nicht ankomme (Innere Sicherheit, Nr. 2/1968, S. 3).

so Die am konkreten Exempel der SRP statuierte Entfernung der Abgeordncten einer illegalisicrten Partei aus den parlamentarischen Volksvertretungen schlug sich über die wiederum generalisierende Formulierung des SRP-Urteils alsbald in der Wahlrechtsgesetzgebung nieder. Damit ist nicht weniger dekretiert und behauptet, als daB die in Art. 38 Abs. I GG aufbewahrte Gewissensfreiheit der Abgeordneten gegenüber demokratisch illegitimen Auftrïgen und Weisungen weder stattfinden dürfe noch könnc. Die mangelhate Realisierung der Gewissensfreiheit der Träger des s freien Mandats a, ein Hauptfaktor des für die Entdemokratisierung des parlamentarischen Systems ursächlichen Geflechts interdependenter Vorgänge, wird sanktioniert; Mandat wird in Herrschatt transformiere.

21 R. Sdnuster (Vgl. Anm. 17, Erstzitat) vergleicht den im politischen Immobilismus der Geselischaft resultierenden ProzeB sehr ansthaulich mit dem Blockierungs- und Zerstörungseffekt einer vollen Fahrt mit ständig angezogener Notbremse, der sich in dem Maße multipliziert, wic blindes Staunen über die zwangsläufige Auflehnung der zwangsiäufig ausgezogenen Opposition gegen das Establishment dessen blindes Zurüdxschlagen zur Folge hat. Nur politische Begabungen vom Range eines Münchbausen - obwohl I go Jahre äler als das andere Geburtstagskind des Jahres 1970, hat er bis heute überlebt - vermöchten sich auch auf und mit dem Ast, den sie sich mit dem schneidigen Streitzug der militanten Demokratie selbst abgesägt haben, mittels der bekannten Shopftehnik in der Schwebe zu halten.

22 So durd die unkricishe Bejahung der Grundgesetzmäßigkeic von $\${ }_{4} 6$ Abs. 3 BVerfGG (vgl. Ridder: KPD-Verbor, S. $\varsigma 2$ f.), die den unauffälligen (und unbemerkt gebliebenen) Sog von der Parteiillegalisicrung (Feststellung der $\gg$ Vertassungswidrigkeita) zum Partei-»Verbota (mit weittragenden Folgen für kollektives Handeln und Sich-äußern der Mitglieder der illeralisicrten Partei) verstärkte (worauf bisher als cinziger W. R. Beyer [vgl. Anm. I6] aufmerksam gemache hat).

s9 Val. Ridder: KPD-Verbot, S. $49 \mathrm{ft}$. 
den atitueller bundesrepublikanischer Deutschlandpolitik gestrichen werden mußte, kann der mit jener Selbstbefristung zum Ausdruck gebrachten Erkenntnis, daß die Fortdauer des KPD-»Verbors « politisch in striktester Abhängigkeit von der Fortdauer der innerdeutschen Konfrontation steht, selbstverständlich keinen Abbruch tun. Umgekehrt: Mit dem Ende dieser Konfrontationsperiode ist politisch das Ende des KPD-"Verbots « gekommen. Indem die Ablösung der *Wiedervereinigungs «-Politik früherer Bundesregierungen, die immer Konfronrationspolitik war, durch eine die deutsche $Z_{\text {weistaatlichkeit akzeptierende, auf }}$ Gesamreuropa bezogene Normalisierungs-, Sicherheits- und Friedenspolitik globalen Übergängen in die Ära der Verhandlungen entspricht, wird auch deurlich, daß die Beendigung des KPD-»Verbots - ein natürlich der bundesrepublikanischen Souveränität unterliegender Vorgang - darüber hinaus für die Stabilisierung des Weltfriedens, der bundesrepublikanische Politik nach Art. 26 GG verfassungsmäßig verpflichtet ist, unerläßlich wird. Fragt man sich, »ob die seit über einem Jahrzehnt in die diplomatische Isolierung der DDR gesteckten Bemühungen nicht heute drohen, zu einem Hemmnis der Bewegungsmöglichkeit der Bundesrepublik selbst zu werden «"4, so deckt man nur die gegebene Parallelität der globalen, der europäischen und der deutschland-politischen Norwendigkeiten auf, denen wiederum bestimmte innenpolitische Notwendigkeiten entsprechen. Die »historische Lage einzusehen «, die das erfordert, mag freilich »nur schwer ankommen*, besonders wenn solche Einsicht sich erst in der Folge schmerzender Einsichten in unsympathische Änderungen globaler Machtbalanzierungen mühsam ihren Weg bahnen kann und hochgezüchtete Emotionen "einer ruhigen Beurteilung der Politik des anderen Teils« und dem Abbau der "gegenseitigen Verketzerung und Bekämpfung « widerstehen ${ }^{25}$. Komme sie indes an, so fällt mit der totalen, aber yorerst noch gratis und ohne Vollzug mit kochendem Wasser zu handhabenden Umtaufe der Bundesrepublik von einem auf die Heimholung der (in Wirklichkeit nie ausgezogenen, sondern allein gelassenen) „Brüder und Schwestern« angelegten europäischen Unruheherd zu einem kraft des "Mitcinander in Deutschland «"26 zentralen Faktor der europäischen Friedensordnung die weitere Erkenntnis des Charakters des KPD*Verbots " als einer versteinerten Verinnerlichung überholter zwischen-deutscher Konfrontation mit einiger Leichtigkeit an.

Das BVerfG hat schließlich, in diesem Punkt ganz konkret formulierend, die "Doktrin des Marxismus-Leninismus" als "Wissenschaftslehre" ausdrücklich nicht in die Reichweite seiner Entscheidung einbezogen ${ }^{27}$ und insofern einen sehr entschiedenen Trennungsstrich zwischen dem Marxismus-Leninismus und dem im SRP-Urteil von ihm festgestellten und zum Ausscheiden aus dem Prozeß der politischen Willensbildung verurteilten makabren NS-»Gedankengut* gezogen. Wegen der ohne triftigen Grund erfolgten doktrinären Verallgemeinerung bei der Darstellung der Rechtsfolgen der konkreten Parteiillegalisierung es wäre übrigens ohnehin vermessen, bei ganzen zwei (sich auch in langer absehbarer Zeit nicht weiter vermehrenden) Fällen des Gebrauchmachens von

-s $U$. Schesıner: Die außenpolitischen Probleme der neuen Bundesregicrung, in: Außenpolitik nach der $\mathbb{V} a \mathrm{hl}$ des 6. Bundestages (Aktuelle AuBenpolitik - Schriftenreihe des Forschungsinsticuts der Deurschen Gesellschaft für Auswärtige Politik, 1969), S. 9 ff. (14).

is Zitatstude nach Soljeuner ebd.

:o Sdjeuner a. a. O., S. I3.

$\therefore$ BVerfGE 5, 145 f.; vgl. auch die in der son den Mirgliedern des BVerfG herausgegebene Sammlung nicht abgedrudiste. jedoch in dem von G. Pfeiffer und H.-G. Strickert herausgerebene Dokumentarwerk $\diamond K$ KD-Prozeß\& wicdergegebene $Z$ wischenerklärung des Senaes: $\diamond$ Als Wissenschattslehre ist die Dokerin des Marxismus-Leninismus nicht Gegenstand des gerichtichen Verfahrens " (3. Bd., r956, S. 583). 
Art. 21 Abs. 2 GG von der Judikatur zu diesem Institut zu sprechen - konnten diese Differenzierung unbeachtet und ihre weittragende Bedeutung verkannt bleiben. Daß der entscheidende Senat damals nicht in der Lage gewesen ist, sich über den modernen Stand der Wissenschaftswissenschaft, z. B. der Kontroverse über den Komplex ihrer w Wertfreiheit « und der auch für nicht marxistisch fundierte Wissenschaft gravierenden Theorie-Praxis-Beziehungen $\mathrm{zu}$ vergewissern, und infolgedessen und zu einer Zeit, als beispielsweise die Publikumswirkung der »Kritischen Theorie" der sog. Frankfurter Schule sich im freundlichen Wiedergutmachungsgewoge auf die Bereicherung des Feuilletons anspruchsvollerer Gazetten beschränkte, zu denkgesetzlich unhaltbaren Aussagen über die »eindeutig bestimmbare Grenze zwischen wissenschaftlicher Theorie und politischem Ziel " gelangt ist ${ }^{28}$, kann hier auf sich beruhen. Wenn auch der vom Senat zugrunde gelegte überholte quietistische Wissenschaftsbegriff (der zwar auch den Schutz von Art. S Abs. 3 GG genießr, auf den dieser Schutz aber nicht beschränkt werden darf, wenn das systemkonstituierende Fundamentalrecht der Meinungsfreiheit nicht durch das opportunistische Ausnahmeregime ausgehöhlt werden soll) unhaltbar ist, so hat der Senat doch, indem er die Wissenschaftsichre des Marxismus-Leninismus "selbstverständlich « unter den Schutz von Arr. S Abs. 3 GG gestellt hat, auch alle die Möglichkeit von »Wertfreiheit " leugnende Wissenschaft im Schurzbereich des Grundrechts belassen. Solche "Idcen " sind keinem Transportverbor innerhalb des Kommunikarionsverbands der Staatsbürger unterworfen. Wie und mit welchem Ergebnis marxistisch-leninistsche "Ideen", die laut BVerfG eine "als solche " freie "Theorie " ausmachen, deren $»$ wissenschaftlicher Wahrheitsgehalt « in der grundgesetzlichen Verfassungsordnung »der Beurteilung eines Gerichts nicht unterliegen « kann, von einer "auf dem Boden" des Marxismus-Leninismus als "wissenschafticher Grundhaltung "stehenden politischen Gruppierung für die Herausbildung politischer Zielvorstellungen verwertet werden, die ssich letztlich in praktischem Handeln niederschlagen ${ }^{29}$, bestimmt sich, wie die sehr verschiedenartige Entwicklung kommunistischer Parteien unter den verschiederten nationalen, geselischaftlichen und politischen Systembedingungen belegt, nach ihrem prinzipiellen Verständnis vom Marxismus als wissenschaftlich fundierter Anleitung zum praktischen Handeln unter den verschiedenen Bedingungen unterschiedlich.

Die Illegalisierung der KPD erfolgte im Hinblick auf ein bestimmtes programmatisches und Handlungsergebnis solcher Umsetzung kombinierter analytischprognostischer Bemühungen. Wird die damals illegalisierte KPD daran gehindert, von der für ihre Anhänger wie für jedermann stets frei zugänglichen $\gg$ Wissenschaftslehre des Marxismus-Leninismus « aus nach einem gewissen Zeitablauf und in einer veränderten politischen Szenerie durch erneute analyrisch-prognostische Bemühungen neue Zielvorstellungen und Handlungsmaximen zu entwickeln - die wegen der inzwischen eingetretenen Veränderungen auch anders ausfallen werden -, so verstößr das auch gegen Art. s Abs. 3 GG. Es kann offen bleiben, ob $»$ die Tarbestände des Art. 5 Abs. 3 und des Art. 2 r Abs. I GG sich... berühren «30. Einschlägig für den Vorgang ist vielmehr die enge Berührung des Tatbestandes von Art. 2 I Abs. I Satz 2 GG mit dem der nicht nur Wissenschaft im Elfenbeinturm schützenden Wissenschaftsfreiheit. Was mir dem nicht einschränkbaren Rückgriff der KPD auf die $\$$ Wissenschaftslehre des Marxis-

29 >Im übrigen... klar zu beroner, daß die Tarbestände des Arr. s Abs. 3 und des Art. 21 Abs. 2 GG sich überhaupe niche berühren (BVerfGE s, r46), kann die state der im übrigen unwichtigen Betonung allein wichtice und erforderliche Begrundung des Betonten nicht ersetzen. 2a Alle zitierten Stellen aus BVerfGE s, its $\mathrm{f}$.

so Vgl. Anm. is. 
mus-Leninismus« geschieht, ist nümlich zugleich der Beginn der Gründung einer im Lichte von Art. 2 I Abs. I Satz 2 GG neuen Partei. Die "Vorstellungsbilder « (d.h. mit dem BVerfG das, w was die Partei sich in ihrer politischen Wirksamkeit im Staate zu erreichen vorgenommen hat ${ }^{31}$ ) dieser in der Entstehung begriffenen und im Entstehungsprozeß von Art. 2 I Abs. 2 Satz 2 GG geschützten neuen Partei können vermöge des formellen Parteienprivilegs nach Art. 2 I Abs. 2 GG, in das die neue Partei hineinwächst, gof. nur wieder in einem entsprechenden Verfahren vor dem BVerfG auf ihre eventuelle „Verfassungswidrigkeit" hin geprüft werden ("verfassungswidrig" ist in der Systematik von Art. 2 I Abs. 2 GG nicht gleichzusetzen mit dem durch die Vorrangstufung von Arr. 20 Abs. 3 GG angesprochenen Komparativ zu "gesetzwidrig “, sondern ist eine vom BVerfG rechtswirksam festgestellte spezifische Qualität einer politischen Partei). Die partielle Identität des Sozialsubstrats der neuen Partei mit dem der alten KPD steht der juristischen Neuqualifikation genau so wenig im Wege wie die Rechtskraft " des KPD-Urteils ${ }^{32}$. Und auch die aus Prestige- oder welchen sonstigen Gründen auch immer erfolgte Eigenfirmierung der in der Neugründung befindlichen Partei als Fortsetzung der illegalisierten KPD vermag an dem Befund nichts zu ändern, daß von Verfassungsrechts wegen die Erarbeitung und Vorlage des Entwurfs 68 ein Vorgang der Parteigründung gewesen ist. Das ist möglicherweise auch von den Gründern der DKP verkannt worden, deren vielleicht im übrigen verständliches Vorgehen die allgemeine Kommunismuskonfusion vermehrt hat und leicht als stillschweigende Anerkennung offizieller und offiziöser maximierender Auslassungen über die Rechtsfolgen des KPDUrteils mißdeutet werden kann.

$\mathrm{Zu}$ den naheliegenden Folgen des beiderseitigen Verkennens der Vorlage des Entwurfs 68 als eines Teilakts einer Parteineugründung im Sinne der grundgesetzlichen Parteiordnung gehör es denn auch, daß die zur Information »berufenen « (und aufgrund ihrer Berufung mobjekrivierenden«) amtlichen Dienste alsbald verlautbarten, die DKP sei in keiner Beziehung müber den Schatten der KPD « gesprungen ${ }^{33}$. Ergebnis: Der Teufelskreis - Neue Partei nur durch neues Programm, neues Programm darf aber nicht von Kommunisten entworfen werden - bleibt fest geschlossen. Die durch das Spiel mit der DKP eingetretene unerträgliche Einbuße an Rechtsstaatlichkeit (Rechtssicherheit) könnte durch den "staatspolitischen Vorteil «, im Hinblick auf das »Verbot BVerfG das Gesicht gewahrt zu haben, nie ausgeglichen werden: Das Grundgesetz gibt jenseits seiner Ordnung keiner Staatsräson Raum; und schlimmer als durch die Mausefallentechnik gegenüber der DKP kann die Optik des Rechtsstaates und damit das Ansehen der Staatsgewalt kaum beeinträchtigt werden.

Der vom LG Flensburg und vom BGH inkriminierte KPD-Entwurf $68^{34}$ enthält übrigens in den analytischen Teilen nichts, was nicht auch in Hunderten und Aberhunderten von wissenschaftlichen (soziologischen, politologischen, zeitgeschichtlichen) Abhandlungen, Büchern, Paperbacks usw., die vielfach (und naturgemäß) zwar differenzierter sind, denen aber das Recht auf Irrtum und Zuspitzung genauso eignet wie dem Entwurf und die, wie den Entwurf, jedermann für irrig und übertrieben zu halten berechtigt ist, an Analyse von Staat und Gesellschaft der Bundesrepublik auf das interessierte Publikum einströmr ${ }^{35}$. Von dem,

31 BVerfGE s, 146.

32 Vgl. hierzu Ridder: KPD-Verbor, S. 40 bis 43.

53 Aktuelle Beiträge zur Innenpolitik, Nr. 7/rg6g ( Die Grundsatzerklärung der DKP vom 23. r. 19694).

s4 Durchgesehen wurde der vom "Initiztivausschuß für die Wiederzulassung der KPD. herausgegebere Undruds (36 Seiten). 
was - mit demselben Recht auf Irrtum und Zuspitzung und demselben Risiko, für irrig und übertrieben gehalten zu werden - zum Lob des wsozialistischen deutschen Staats « namens DDR an Tatsachen vorgetragen wird, hat unliingst ein langjähriger Berater mehrerer Bundesregierungen mindestens einen erheblichen Teil wohl für so bedeutend gehalten, daß er, ihr allgemeines Bekanntsein voraussetzend, »Achtung vor der großen Leistung der Bevölkerung wie der Führung in der DDR, die wireschaftlich bedeutende Ergebnisse erzielt hat «, fordert ${ }^{36}$. Damit will er auch die weitere Annäherung der deutschen an koreanische oder vietnamesische Spaltungszustände aufhalten - in Südkorea ist bercits der Bericht über die Fortschritte der Elektrifizicrung Nordkoreas zuchthauswürdig -, dic kräftig gefördert wird, wenn die "Idealisierung " von DDR-Verhältnissen Rechrsnachteile hervorruft. Was schließlich das Programmatische im engeren Sinne des Wortes angeht, läßt sich diesem Entwurf, der trotz seines Wortreichtums manche empfindlichen sachlichen Lücken ${ }^{37}$ aufweist und wegen diverser lyrischer Einlagen und antiquierter Beschwörungsformeln in der Bundesrepublik nicht übermäßig attraktiv wirken dürfte, lediglich die pointierte Forderung nach besserer Wahrung des Grundgesetzes und Ausschöpfung seiner Möglichkeiten für eine zugleich sozialistische und demokratische Politik entnehmen ${ }^{38}$.

Die vom BGH akzeptierte Qualifizierung des Entwurfs als sverfassungsfeindlich « verdeutlicht zweierlei. Erstens, daß der in der Rechtsprechung praktizierte irrationale Antikommunismus nach wie vor Verinnerlichung und Funktion des binnendeutschen "Kalten Krieges« ist; denn die als nbewußt negativ, überspitzt " und "unzutreffend " bezeichneten Tatsachenbehauptungen des Entwurfs greifen laut LG Flensburg »die freiheitliche demokratische Grundordnung [nur!] an, wenn die zum Teil wahrheitswidrig dargestellten Verhältnisse in der DDR gegenübergestellt werden «. Z weitens, daß die wfreiheitliche demokratische Grundordnung", die nur ein Gebot des Grundgesetzes sein kann, sich unter der Schwerkraft der politischen Entwidklung in der Judikatur des BGH definitiv mit dem Status der gesellschaftlichen Verhältnisse, dem Status tel quel, identifiziert hat ${ }^{30}$.

Das BVerfG, dessen im Zusammenhang einer um demokratische Orientierung

33 Diese Disziplinen haben sich, wenn ihnen auch เ.. a. die mögliche emanzipatorische Funktion von (verfassungskonformem) Recht weithin noch nicht aufgegnngen ist, doch immerhin selbst soweis emanzipiert, daß sie allmählich aufhören, seiner dankbaren Umwelt das, was diese gern hörtex, zu suggerieren ( $U$. Jaeggi: Machs und Herrschaft in der Bundesrepublik, Reihe - Informationen zur Zeita der Fischer-Bücherei ror4 [1969]).

38 Scheuner a. 2. O. S. 14.

37 Zur Kritik von linksa vgl. erwa E. Fried: Zum KPD-Programm-Entwurf, Kürsbiskern 3/1968, S. $440 \mathrm{ff}$. (443), der vor allem die Auseinandersetzung mit den . Fehlern und Deformationen der Stalinzeita und den Problemen der Schüler und Studenten vermißr, jedoch mit der Bemängelung des Fehlens von Parteistatucen und Aussagen über die Parteistruktur übersieht. daß die Vorlage eines Programmentwurfs bloßer Teil eines mehrphasigen Parteigründungsvorgangs sein kann; sowic ASO (Arbeitsgern. Sozial. Opposition): Stellungnahme zum Programmentwurf der KPD, Sozialistische Hefte, 7. Jg. Heft \& (August 1968), S. 465 ff., wo dic unpolicische Terminologie der programmatischen und die Unschärfe der analycischen Teile des Entwurfs angegriffen wird.

${ }^{8}$ Damit ist die - auch durd das tatsädhliche Verhalten der bundesdeutschen KPD-Anhänger angesidhts der Demonstrationsunruhen der letzten Jahre bestätigte - europaweite Orientierunc kommunistischer Parteicn auf den sfriedlichen Ubergang zum Sozialismusa (vgl. für die KRD etwa Parteitag r 963 der KPD, Protokoll, S. 463; Bericht des Politbüros auf der 3. Tagung des ZK, Wissen und Tar, Sondernummer Juni 1964, S. Is; +. ZK-Tagung, Wissen und Tar. Hett $\sqrt{2}$, 1969 , S. 3 I f.; 7. ZK-Tagung [Ablehnung einer DDR-Kopie für die Bundesrepublik] Wissen und Tat. Hcft $5 / 6,1966$, S. 20 f.) bestätigt.

3s Auch terminologisch tritt dieser Abstieg bis zum Boden bereits gelegentlich in Erscheinung, ohne daß die Praxis noch fähig wäre, vor solcher Selbstoffenbarung zu erschrecken. So wird in der Anklageschrift bei dem LG Hamburg v. 11. 7. 1969 gegen Röhl (1+1 Js 1309/68) dem Angeklagten das Handlungsziel oder Aushöhlung ... der bestehenden Verfassungswirklichkeir [!] * zur Last gelegt. 
bemühten Abwägung gebrauchten Formulierungen wörtlich übernommen und dabei rücksichtslos verfälscht worden sind ${ }^{40}$, hat in einer fatalen Serie neuer Beschlüsse vom Januar I $969^{41}$ unter Zurückweisung partiell demokratiedienlicher Hilfsangebote des Bundesministers der Justiz 2 im Bann von $\$ 46$ Abs. 3 BVerfGG den weiterhin politisch tätigen einzelnen früheren KPD-Angehörigen auch jegliches bloß objektiv auf die KPD »organisationsbezogenes « Handeln untersagt, das mit einem "unmittelbaren Förderungseffekt " für die für verfassungswidrig erklärte Partei verbunden ist $^{43}$. Das »Parteiverbot soll « aber »nur objcktive Gefahren abwehren * ${ }^{44}$. Daß ein bestimmter Tätigkeitsinhalt einer bestimmten Partei in einer bestimmten Situation als "objektive Gefahr « angesehen worden ist, rechtfertigt aber nicht den Schluß, daß jede Tätigkeit des Parteisubstrats in jeder Situation eine sobjektive Gefahr « darstellt. Ein solcher Schluß macht anstelle des Tatigkeitsinhalts, aufgrund dessen die Partei illegalisiert wurde, das Illegalisiert-(worden-)sein der Partei zum Kriterium einer gegenwärtigen „objekriven Gefahr «"5. Er läuft auf den ebenso untauglichen wie undemokratischen Versuch hinaus, künftige politische Entwicklungen einzumauern, und sollte sich durch seine bare Unlogik selbst erledigen.

Es kann und muß also auch von dem Zentralpunkt der Argumentation jener Beschlußserie des BVerfG, die sich im einzelnen mit den tautologischen Sequenzen und petitiones principii fremder und anderer eigener Judikate verquickt und von der man hoffen möchte, daß sie eine Schlußserie bleibr, der Rückweg in den demokratischen und rechtsstaatichen Verfassungshafen angetreten werden, der dem ausgelaufenen Staatsschiff der Bundesrepublik durch die Aufrüstung zur "Militanz " infolge des Koreaschodks so lange außer Sicht geraten ist. Nach dem dort geltenden Parteienregime ist es zwar statthaf, daß eine politische Partei auf Antrag der unter dem Opportunitätsprinzip ${ }^{46}$ antragsbefugten Stellen von dem allein zur Entscheidung befugten Gericht illegalisiert wird, wenn und weil ihre Tätigkeit nach der übereinstimmenden Auffasung von Antragsteller und Gericht eine "objektive Gefahr« für den Verfassungsbestand darstellt, kann jedoch die in immer engeren Spiralen scheinlogischer Deduktionen niederkommende despotische Eulenspiegelei nicht rechtens sein, nach der eine Partei deswegen aktuell eine "objektive Gefahr « darstellt, weil sie in einem solchen Verfahren illegalisiert worden ist, und müssen infolgedessen die Rechtsfolgen der Illegalisierung da ihre Grenze finden, wo diese Eulenspiegelei beginnt.

40 So gelangte, um nur ein Beispiel zu nennen, auch das KPD-Urteil des BVerfG zu einer -Gleichwertigkeic von Art. 5 und Art. 2 r Abs. 2 GG, dies aber nur, um die (selbstrerständlich zu verneinende, aber damals unter dem Einfluß von Naturrechtsepigonen noch modische) Frage zu verneinen, ob Art. 2 r Abs. 2 GG selbst (eine Bestimmung aus dem ersten unrevidierten Gúf des Grundgeserzes) nverfassungswidrig a sein könne. Das geschah, wie die einschlägige Argumentation erweist (BVerfGE 5, 136 ff.), in klarer Erkenntnis des Ausnahmeregimecharakters von Art. 2 r Abs. 2 GG, der nicht in Zweifel gezogen werden sollte. Aus der vom BVerfG gemeinten Gleichrangigkeit in der Geltungsebene des Verfassungsrechts wurde in nicht mehr zu zählenden Entscheidungen von Strafgerichten jene Gleichwertigkeit , die in jedem konkreten Einzelfall Uberwertigkeit des Ausnahmeregimes gegenüber der demokratischen Normalitz̈t bedeuter.

41 BVerfGE 25,44 bis ror.

42 Stellungnahme zu vier Verfassungsbeschwerden früherer Angehöriger der KPD gegen strafgerichtliche Verurteilungen v. 9. S. 1968 (r004 E [1687] - 129/68).

13 BVerfGE 25,58 .

44 Ebd.

45 Wer den endlosen Paraphrasen dieser Umstülpung nicht aufsitzt, wird, wie das am KPD. Urteil beteiligt gewesene frühere Mirglied des Ersten Senats des BVerfG Dr. Soboltissek in einer Podiumsdiskussion des ZDF vom 17.8. 1967, zu der nüchternen Feststellung gelangen, daB die KPD unter den Umstanden der letztvergangnen Jahre nicht illegalisiert worden wäre.

48 So zutreffend und mit aller Deutlidukeit gegenüber dem Vortrag der Bundesregierung der Zwisciensprudi des KPD-Urreils (KPD-ProzeB, Dokumentarwerk, S. 583). 
Diese Grenzen sind einigen der Grundgesetzväter gegenwärtig gewesen ${ }^{17}$. Am Rande des Weges zu ihrer Wiederentdedkung, den die Sehenden und Wissenden längst hätten gehen müssen, aber nicht gegangen sind, weil das Schweigen so oft der bequemere Teil der Wissenschaft ist, wird sich der Schutt einer bisher kaum ernsthaft auf ihre Grundgesetzmäßigkeit hin geprüften straf-, vereins- usw. rechtlichen Folgegesetzgebung ${ }^{48}$ und der unkonkret vom Untergang der Reichsrepublik als Folge »der Zulassung staatsfeindlicher Parteien «"9 fabulierenden staatsrechtlichen Kompendienliteratur ${ }^{50}$ häufen müssen, wenn die Möglichkeit wiedergewonnen werden soll, in freier Auseinandersetzung mit den ernstzunehmenden Versuchen demokratischer Staatsgestaltung unseres Jahrhunderts ${ }^{51}$ zu entscheiden, ob die vorhandenen - nicht selbstzweckhaften - verfassungsmäßigen Sicherungen des »demokratischen und sozialen « Staats (noch) ausreichen (oder wieder ausreichend gemacht werden können), um die stieferen Probleme sozialer Gerechtigkeit ${ }^{50}$ zu lösen. Darum geht es auch bei der nun anstehenden neuen Entscheidung des LG Flensburg in dem Einziehungsverfahren betreffend den KPD-Programm-Entwurf 1968.

Eine Erweiterung des in erster Instanz erfolgten bescheidenen Durchbruchs zur Verfassung hat der BGH allerdings mit einer (in sich schlüssigen) prozessualen Volte zu verhindern versucht: $\mathrm{Da}$ die "Darlegungen des Landgerichts über den verfassungsfeindlichen Inhalt der Schriften... nicht zu den tragenden Gründen des angefochtenen Urteils « gehörten und daher möglicherweise weniger ausführlich seien, »wie es der Fall wäre, wenn die Entscheidung auf ihnen beruhte «, *könnte eine Sachentscheidung des Senats allein auf Grund der bisherigen Urteilsfeststellungen zu einer Beeinträchtigung der verfahrensmäßigen Rechte der Einziehungsbeteiligten führen. « Dieses Musterbeispiel von zweckentfremdend"eigenständiger « Prozeßrechtshandhabung, die über den prätendierten Schutz von Verfahrensrechten gegen die materiellen Rechte der Beteiligten zielt, indem sie sich des weiteren mit einer penetranten Suggestion des gewünschten Ergebnisses verbindet, ist in der Presse bündig beim Namen genannt worden: "Der Dritte Strafsenat des Bundesgerichtshofs ordnete eine neue Begründung über die Einziehung der 60000 Exemplare des KPD-Programmentwurfs vor einem anderen Gericht an «53.

47 Der Abg. Dr. Debler bezeichnete es im Parlamentarischen Rat als die Hauptfolge der Illegalisierung, daß die Partei sich niche mehr an Wahlen beteiligen kanne (JOR NF I [I95I], S. 208).

48 die überspielt, daß nach der besatzungsrechtlichen Beseitigung des gesamten politiscaen Serafreches das Grundgeserz mir Arr. I43 a. F. die Chance bor und den Aufrag gab, undemokratische Stazesräson voll und ganz durch Verfassungsräson zu ersetzen.

49 Maunz-Dürig-Hegzog: Grundgesetz, Rd.-Nr. Ior zu Art. 21.

50 In einem Parallelzusammenhang (Art. s und I8 GG) falsch akzentuierend, weil die schon angelaufene Folgegeserzgebung zu Art. 2r Abs. 2 GG niche berücksichtigend und Art. I 8 und 21 Abs. 2 GG mit der damaligen Literatur als Verstärkung des tradierten > Staatsschutzes* wertend, insbesondere auch $H$. Ridder in dem 1952 entworfenen Beitrag - Meinungstreiheit* (Die Grundrechie, Bd. II [1954], S. 243 ff. [285 f..]).

31 Vgl. C. B. Macpherson: Drei Formen der Demokratie (1967).

$52 \mathrm{Vgl}$. Ridder: KPD-Verbot, S. 27, mit Zitat Scheuner in Anm. 46.

37 Süddeutsche Zeitung 7. 19.2.1970 (-Keine politische Handlungsfreiheir für die verbocene KPD*). 